\title{
Dispersiones Acuosas de Poliuretano Bloqueado: Una Alternativa como Agente Curtiente
}

\author{
José Luis Mata-Mata, Diana Mendoza Olivares, Diego Ismael Alvarado Castro, Fernando de Jesús Amézquita López \\ Facultad de Química, Universidad de Guanajuato, México
}

\begin{abstract}
Resumen: Se sintetizaron y se evaluaron como agentes curtientes de pieles vacunas dos tipos de agentes de reticulación base poliuretano bloqueado. Se presentan los resultados de la síntesis de dispersiones acuosas de poliuretano bloqueado (DPU's), basadas en prepolímeros a partir de la reacción de los diisocianatos alifáticos $\mathrm{H}_{12} \mathrm{MDI}$ (4,4' metilenbis-ciclohexilisocianato) y HDI (hexametilendiisocianato) con polioles base poliéter óxido de etileno de peso molecular 1 y $2 \mathrm{KDa}$, en una relación molar isocianato/oxhidrilo $(\mathrm{NCO} / \mathrm{OH})$ de $4: 1$ y $6: 1$ a $100{ }^{\circ} \mathrm{C}$ por 2 y 4 horas. En una segunda reacción, los grupos isocianato (NCO) libres del prepolímero se bloquearon con una solución acuosa de $\mathrm{NaHSO}_{3}$ ó $\mathrm{Na}_{2} \mathrm{~S}_{2} \mathrm{O}_{5}$ al $40 \%$ p/p. Los prepolímeros se caracterizaron mediante espectroscopia IR y RMN de ${ }^{1} \mathrm{H}$. El tanto por ciento de isocianato libre en el prepolímero, así como el bloqueo de dichos grupos, se determinaron por espectroscopía IR. La evaluación de la capacidad curtiente de las DPU's se determinó de acuerdo a la estabilidad térmica de la piel (temperatura de desnaturalización), mediante Calorimetría Diferencial de Barrido (DSC) y a la determinación del índice de reticulación de las DPU's con la colágena de la piel mediante un ensayo con ninhidrina.
\end{abstract}

Palabras-clave: Dispersiones acuosas de poliuretano bloqueado (DPU's), agentes curtientes, reticulación.

\section{Aqueous Dispersions of Blocked Polyurethane: An Alternative as Tanning Agents}

\begin{abstract}
Two types of crosslinking agents blocked polyurethanes were synthesized and evaluated as tanning agents of bovine skins. The results of the synthesis of aqueous dispersions of blocked polyurethane (DPU's) are presented. They were based on prepolymers from the reaction of the aliphatic diisocyanates $\mathrm{H}_{12} \mathrm{MDI}$ 4,4'-methylenebis(cyclohexyl isocyanate) and HDI (hexamethylene diisocyanate) with HO-polyols such as polyethylene oxide of molecular weight 1 and $2 \mathrm{kDa}$. The molar $\mathrm{NCO} / \mathrm{OH}$ ratio was fixed in $4: 1$ or $6: 1$, at $100{ }^{\circ} \mathrm{C}$ and 2 or 4 hours for the reaction time. In a second reaction, the prepolymer containing terminal $\mathrm{NCO}$ groups were blocked by reaction with an aqueous $\mathrm{NaHSO}_{3}$ or $\mathrm{Na}_{2} \mathrm{~S}_{2} \mathrm{O}_{5}$ solution to $40 \% \mathrm{w} / \mathrm{w}$. The obtained prepolymers were characterized by IR and ${ }^{1} \mathrm{H}$ NMR. The $\%$ of free isocyanate (NCO) in the prepolymer as well as the blockade of these groups were determined by IR. The evaluation of the tanning capacity of the DPU's was determined according to the thermal stability of the skin (thermal denaturalization), through the differential scanning calorimetry analysis (DSC). The index of crosslinking of the DPU's with the collagen of the skin was determined with the nynhidrin essay.
\end{abstract}

Keywords: Aqueous dispersions of blocked polyurethane (DPU's), tanning agents, crosslinking.

\section{Introduccion}

Las sales de cromo han sido ampliamente utilizadas como agentes curtientes, aunque muchas veces el cuero resultante requiere ser recurtido con curtientes vegetales o sintéticos. Recientemente las leyes que regulan el impacto ambiental en todo el mundo, han sugerido reemplazar el cromo como agente curtiente y remover todas las trazas de cromo que se encuentran en los residuos de tenerías. Esto ha promovido la investigación en la búsqueda de otros curtientes, como alternativa al proceso de curtido con cromo. Una posibilidad es el desarrollo de agentes curtientes, capaces de reemplazar completamente al cromo requerido para curtir. Existen varios compuestos orgánicos bifuncionales que son capaces de reticular químicamente las cadenas moleculares de la colágena, formando enlaces covalentes, y que además son potencialmente capaces de servir como agentes curtientes. A nivel industrial sólo el formaldehído y el glutaraldehído son utilizados como agentes de reticulación molecular con la colágena ${ }^{[1]}$. Probablemente el número, tamaño y localización de la reticulación así como los cambios en la carga electrostática y carácter hidrofílico-hidrofóbico de la colágena juegan un papel determinante en las propiedades del cuero resultante.

Los diisocianatos han sido usados en la curtición del cuero por su capacidad de introducir reticulaciones covalentes. Sin embargo, esta curtición tiene la desventaja de la alta toxicidad de los isocianatos, el uso de disolventes orgánicos y la pérdida del agente curtiente; por competencia de la hidrólisis que ocurre durante el tratamiento acuoso. Petersen $^{[2]}$, ha descrito un método para la formación de iso-

Autor para correspondência: José Luis Mata-Mata, Facultad de Química, Universidad de Guanajuato, Noria Alta s/n; Caixa Postal 36050, Guanajuato, México, E-mail: matamm@quijote.ugto.mx 
cianatos modificados (carbamoilsulfonatos) por reacción de un isocianato con el ión bisulfito:

$$
\mathrm{RN}=\mathrm{C}=\mathrm{O}+\mathrm{HSO}_{3}^{-} \rightarrow \mathrm{RNH}-\mathrm{CO}-\mathrm{SO}_{3}^{-}
$$

Si el ión bisulfito es acompañado de un catión altamente soluble, por ejemplo el sodio, se forma una sal de carbamoilsulfonato de sodio soluble en agua:

$$
\mathrm{RNCO}+\mathrm{NaHSO}_{3} \rightarrow \mathrm{RNHCOSO}_{3}^{-}+\mathrm{Na}^{+}
$$

Algunas de las ventajas que presentan los diisocianatos modificados no sólo son su alta solubilidad en agua, sino además su alta resistencia a la hidrólisis en solución ácida o neutra y su reactividad con grupos aminos para formar uretanos:

$$
\mathrm{RNHCOSO}_{3} \mathrm{Na}+\mathrm{R}^{\prime} \mathrm{NH}_{2} \rightarrow \mathrm{RNHCONHR}^{\prime}+\mathrm{NaHSO}_{3}
$$

De tal manera que los diisocianatos modificados podrán reticularse molecularmente con los grupos aminos libres de la colágena ${ }^{[3]}$. Kenyon ${ }^{[4]}$ y col. reportaron evidencias de reticulación molecular entre diisocianatos y la colágena. Cater ${ }^{[5]}$, reportó como agentes curtientes a los diisocianatos modificados (carbamoilsulfonatos) preparados a partir de tolilendiisocianato (TDI) y hexametilendiisocianato (HDI). Sin embargo, encontró que los reticulados moleculares formados eran relativamente pocos. Probablemente debido a que las reacciones fueron llevadas al cabo en medio ácido, donde presumiblemente los grupos amino terminales se encuentran en su forma protonada y no en la forma amino reactiva. Por estudios realizados posteriormente por Milligan ${ }^{[6]}$, usando los mismos reactivos que Cater, llegó a la conclusión de que los isocianatos modificados sólo pueden ser usados como agentes recurtientes para cueros curtidos al cromo. Sin embargo, el mismo autor recomienda continuar con la investigación para usar los isocianatos modificados como curtientes principales.

Los curtidos con isocianatos han sido investigados desde los 40's, del siglo pasado, y aunque fue desarrollado un producto por Bayer; nunca fue producido a escala industrial debido a su toxicidad. Más tarde, Traübel nuevamente investigó sobre la posibilidad de curtir pieles usando isocianatos y sus derivados de bajo peso molecular en medio acuoso. Pero debido a que los isocianatos con grupos aromáticos son cancerígenos éstos fueron evitados, y sólo se estudiaron isocianatos alifáticos de bajo peso molecular como agentes curtientes. Para ello, Traübel ${ }^{[7]}$, hizo reaccionar parcialmente los isocianatos con alcoholes y bisulfito de sodio para disminuir su toxicidad e incrementar su solubilidad en agua.

Los toxicólogos recomiendan no trabajar con isocianatos por su carácter cancerígeno. No obstante, si se bloquean ambos grupos isocianato se elimina la toxicidad del compuesto y con ello el riesgo en su utilización. Posteriormente durante el proceso de curtido se regeneran dichos grupos para interactuar con la colágena de la piel y actuar como agentes curtientes.

Los isocianatos son capaces de reaccionar con los grupos hidroxilos para formar un uretano meta estable. Éste, bajo condiciones estables, puede generar nuevamente el isocianato y el grupo hidroxilo. Por su forma de reaccionar, este tipo de uretanos meta estables es conocido como diisocianatos bloqueados (prepolímeros). Este tipo de reacciones químicas es usada en la industria textil desde hace más de 30 años ${ }^{[8]}$.

De acuerdo a los estudios reportados en la literatura, es posible la curtición de pieles utilizando diisocianatos modificados químicamente. Para ello es recomendado bloquear los grupos isocianato con la intención de disminuir su toxicidad. Sin embargo, se requiere de una mayor investigación, motivo por el cual se planteó el presente proyecto. Se sintetizaron prepolímeros a partir de la reacción de los diisocianatos 4,4' metilenbis-ciclohexilisocianato $\left(\mathrm{H}_{12} \mathrm{MDI}\right)$ y hexametilendiisocianato (HDI), con polioles base poliéter de $1 \mathrm{y}$ $2 \mathrm{KDa}$ en una relación molar $\mathrm{NCO} / \mathrm{OH}(4: 1)$ a $100{ }^{\circ} \mathrm{C}$ a 2 y 4 horas. En una segunda reacción, los grupos diisocianato libres del prepolímero se bloquearon con una solución acuosa de $\mathrm{NaHSO}_{3}$ o $\mathrm{Na}_{2} \mathrm{~S}_{2} \mathrm{O}_{5}$ al $40 \%$ p/v. Lo que permitió la formación de una dispersión de diisocianato bloqueado (DPU). Los prepolímeros se caracterizaron mediante espectroscopia de IR y RMN de ${ }^{1} \mathrm{H}$. El tanto por ciento de isocianato libre (NCO) se determinó por espectroscopia IR.

Posteriormente, se evaluó la capacidad como agente curtiente de los prepolímeros obtenidos. Se llevó al cabo el proceso de penetración y fijación del agente curtiente a escala laboratorio para determinar las condiciones de reacción más favorables. Para evaluar la capacidad curtiente del prepolímero, evaluamos la estabilidad de la piel mediante Calorimetría Diferencial de Barrido (DSC), así como el índice de reticulación determinado mediante la reacción de los grupos aminos libres de la colágena de la piel y la ninhidrina.

\section{Metodologia}

\section{Reactivos utilizados}

4,4-metilen-bis(ciclohexilisocianato) (Vestanat $\mathrm{H}_{12} \mathrm{MDI}$, Degussa), hexametilendiisocianato (HDI Luxanate Aliphatic Isocyanate HM, Lyondell), Polioles base óxido de etileno de peso molecular de 1 y 2 kDa (PEG-1000, PEG-2000), bisulfito de sodio $\left(\mathrm{NaHSO}_{3}\right)$, metabisulfito de sodio $\left(\mathrm{Na}_{2} \mathrm{~S}_{2} \mathrm{O}_{5}\right)$, cloroformo deuterado $\left(\mathrm{CDCl}_{3}\right)$ y cloroformo $\left(\mathrm{CHCl}_{3}\right)$ de Sigma-Aldrich.

\section{Mediciones}

Los espectros de IR se obtuvieron con la técnica de espectroscopia infrarroja, utilizando un espectrofotómetro de transformada de Fourier modelo 1620 series 1600 de Perkin Elmer, en un rango de exploración de 4000 a $650 \mathrm{~cm}^{-1}$ con accesorio de ATR de seleniuro de zinc ( $\mathrm{ZnSe}$ ) en solución de cloroformo.

El tanto por ciento de isocianato libre (NCO) de los prepolímeros se determinó por espectroscopia IR mediante curvas de calibración para $\mathrm{H}_{12} \mathrm{MDI}$ y $\mathrm{HDI}$ en un rango de concentración $1 \% \mathrm{p} / \mathrm{p}$ al $10 \% \mathrm{p} / \mathrm{p}$, en $\mathrm{CHCl}_{3}$. Las soluciones de prepolímero se prepararon al $5 \%$ p/p y la concentración de NCO libre se calculó en la curva de calibración. 
Los espectros de RMN de ${ }^{1} \mathrm{H}$ fueron obtenidos en solución a temperatura ambiente en un equipo Varian Gemini 200 (200 MHz utilizando $\mathrm{CDCl}_{3}$ como disolvente). El desplazamiento químico $(\delta)$ en los espectros fue referenciado respecto a la señal del disolvente a $\delta 7,27 \mathrm{ppm}$.

La temperatura de desnaturalización de la piel se determinó por Calorimetría Diferencial de Barrido (DSC), en un calorímetro Mettler Toledo DSC822 a una velocidad de calentamiento de $10{ }^{\circ} \mathrm{C} / \mathrm{min}$ en un intervalo de 40 a $140{ }^{\circ} \mathrm{C}$.

El índice de reticulación fue determinado mediante la reacción de los grupos amino libres en la colágena de la piel y la ninhidrina (2,2-dihidroxi-1,3-indandiona). La ninhidrina es un potente agente oxidante y lleva a la desaminación oxidativa de grupos $\alpha$-aminos. La reacción entre los grupos aminos primarios de la lisina ó hidroxilisina en la colágena y la ninhidrina forma un compuesto azul violeta (azul de Ruhemann) que tiene una absorbancia máxima en $566 \mathrm{~nm}$. El índice de reticulación es indicativo del tanto por ciento de grupos amino bloqueados. La absorbancia se leyó a $566 \mathrm{~nm}$ en un espectrofotómetro Perkin-Elmer Mod. Lambda 40.

\section{Preparación del prepolímero}

En un matraz balón de $100 \mathrm{~mL}$ mezclar $2 \mathrm{~mL}$ de $\mathrm{H}_{12} \mathrm{MDI}$ $(0,0081 \mathrm{~mol})$ con 2 gramos del PEG-1000 ( $0,002 \mathrm{~mol})$ en una relación molar de $4: 1$, calentar a $100{ }^{\circ} \mathrm{C}$ durante 4 horas con agitación constante. Se tomaron muestras a las 2 horas y a las 4 horas, para su análisis por IR y evaluar el tanto por ciento de NCO libre. El procedimiento para preparar el prepolímero de HDI fue similar.

\section{Caracterización del prepolímero}

Prepolímero con HDI. IR. (Solución en $\mathrm{CHCl}_{3}$ ) (Figura 2): $3326 \mathrm{~cm}^{-1}\left(\mathrm{v}_{\mathrm{N}-\mathrm{H}}\right), 1514 \mathrm{~cm}^{-1}\left(\delta_{\mathrm{NH}} \mathrm{yv}_{\mathrm{C}-\mathrm{N}}\right), 2930 \mathrm{~cm}^{-1}-2852 \mathrm{~cm}^{-1}$ $\left(v_{\mathrm{C}-\mathrm{H}}\right), 2267 \mathrm{~cm}^{-1}\left(v_{\mathrm{NCO}}\right), 1708 \mathrm{~cm}^{-1}$ ( $v_{\mathrm{CO}}$ de amida).

${ }^{1} \mathrm{H} \quad \mathrm{RMN}\left(200 \mathrm{MHz}, \mathrm{CDCl}_{3}, \mathrm{ppm}\right) ; \quad \delta=1,5$ (-NH- $\left.\mathrm{CH}_{2} \mathrm{CH}_{2} \mathrm{CH}_{2} \mathrm{CH}_{2} \mathrm{CH}_{2} \mathrm{CH}_{2}-\mathrm{NH}\right), 4,2\left(\mathrm{O}-\mathrm{CH}_{2} \mathrm{CH}_{2}-\mathrm{O}-\right), 3,3$ (-NH- $\left.\mathrm{CH}_{2} \mathrm{CH}_{2} \mathrm{CH}_{2} \mathrm{CH}_{2} \mathrm{CH}_{2} \mathrm{CH}_{2}-\mathrm{NH}\right), 4,8\left(-\underline{\mathrm{NH}}-\mathrm{CH}_{2} \mathrm{CH}_{2} \mathrm{CH}_{2}\right.$ $\mathrm{CH}_{2} \mathrm{CH}_{2} \mathrm{CH}_{2}-\mathrm{NH}$ ).

Prepolímero con $\mathrm{H}_{12} \mathrm{MDI}$. IR, (solución en $\mathrm{CHCl}_{3}$ ) (Figura 3): $3320 \mathrm{~cm}^{-1}\left(\mathrm{v}_{\mathrm{N}-\mathrm{H}}\right), 1514 \mathrm{~cm}^{-1}\left(\delta_{\mathrm{NH}} \mathrm{yv}_{\mathrm{C}-\mathrm{N}}\right)$, $2930 \mathrm{~cm}^{-1}-2855 \mathrm{~cm}^{-1}\left(v_{\mathrm{C}-\mathrm{H}}\right), 2263 \mathrm{~cm}^{-1}\left(\mathrm{v}_{\mathrm{NCO}}\right), 1715 \mathrm{~cm}^{-1}\left(\mathrm{v}_{\mathrm{CO}}\right.$ de amida).

${ }^{1} \mathrm{H}$ RMN (200 MHz, $\left.\mathrm{CDCl}_{3}, \mathrm{ppm}\right) ; \delta=0,7-2,1\left(\mathrm{CH}_{2} \mathrm{de}\right.$ los ciclohexanos), 4,1 ( $\left.\mathrm{OCH}_{2} \underline{\mathrm{CH}}_{2} \mathrm{O}-\right), 4,8\left(-\underline{\mathrm{NH}}-\mathrm{C}_{6} \mathrm{H}_{10}\right), 3,8$ $\left(\mathrm{C}_{6} \mathrm{H}_{10}-\mathrm{CH}_{2}-\mathrm{C}_{6} \mathrm{H}_{10}\right)$.

\section{Bloqueo de los grupos isocianatos}

El prepolímero formado se hizo reaccionar con una solución de bisulfito o metabisulfito de sodio al $40 \% \mathrm{p} / \mathrm{p}$ en agua $\left(1,685 \mathrm{~g}\right.$ de $\mathrm{NaHSO}_{3}$ en $2,5 \mathrm{~mL}$ de $\left.\mathrm{H}_{2} \mathrm{O}\right)$ para formar la dispersión de poliuretano bloqueado (DPU's) el cual presenta una excelente solubilidad en agua.

Esta solución se adiciona lentamente al prepolímero a la temperatura de reacción de $60^{\circ} \mathrm{C}$. Posteriormente se disminuye la temperatura hasta $40{ }^{\circ} \mathrm{C}$ y se mantiene la agitación por
45 minutos. Finalmente se agregan $4,1 \mathrm{~mL}$ agua $(0,228 \mathrm{~mol})$ y se deja a temperatura ambiente con agitación constante por 8 horas.

Posteriormente se evaluó la estabilidad de la dispersión de poliuretano en reposo a temperatura ambiente. El análisis de la estabilidad del prepolímero formado se realizó por espectroscopia IR.

\section{Evaluación del efecto curtiente de las DPU's}

Las pruebas de curtición se realizaron en viales con agitación a $25 \mathrm{rpm}$. Se partió de una piel depilada, dividida y desencalada (piel en tripa). Las muestras de piel $(5 \mathrm{~cm} \times 5 \mathrm{~cm})$ con un $\mathrm{pH}=8$, se lavaron usando $100 \%$ de agua $\left(30^{\circ} \mathrm{C}\right)$ con relación al peso de la piel, con agitación durante 20 minutos. Posteriormente, el baño fue drenado y la curtición se realizó utilizando $50 \% \mathrm{p} / \mathrm{v}$ de agua $\left(30{ }^{\circ} \mathrm{C}\right), 5$ a $15 \%$ v/v de la dispersión de poliuretano (DPU's). Los viales se mantuvieron en agitación por 90 minutos; el pH del baño y la piel fue de 6,5. A continuación se adicionó el $1 \%$ p/p de $\mathrm{MgO}$ y se agitó 60 minutos. Posteriormente, se agregó otro $1 \%$ p/p de $\mathrm{MgO}$ y se dejó en agitación 8 horas. El pH final de la piel fue de 7,5 a 8,0. Se drenó el baño y se lavaron las pieles con agua.

Se evaluó el efecto del pH en el proceso de curtido. Además, el efecto de un aceite sulfitado y el de un recurtiente fenólico en el proceso.

La determinación de la estabilidad térmica se realizó sobre el cuero húmedo. La determinación del índice de reticulación se realizó sobre el cuero seco.

\section{Determinación de la temperatura de desnaturalización por DSC}

La temperatura de desnaturalización de la piel se determinó por DSC, donde muestras entre 5 y $9 \mathrm{mg}$ en estado hidratado se calentaron a una velocidad de calentamiento de $10{ }^{\circ} \mathrm{C} / \mathrm{min}$ en un intervalo de 40 a $140{ }^{\circ} \mathrm{C}$. La temperatura de desnaturalización fue considerada como la temperatura máxima al pico en el termograma obtenido.

\section{Determinación de índice de reticulación}

Aproximadamente, a $10 \mathrm{mg}$ de piel seca se le adicionaron $3 \mathrm{~mL}$ de una solución de ninhidrina al $2 \%$ p/v (en solución tamponada de citrato/ácido clorhídrico de $\mathrm{pH}=4$ ). La mezcla se calentó por 10 minutos en un baño de agua a $\sim 100^{\circ} \mathrm{C}$. Finalmente, la solución se dejó enfriar a temperatura ambiente, se dejó en reposo 60 minutos y se leyó la absorbancia a $566 \mathrm{~nm}$ en un espectrofotómetro Perkin- Elmer lambda 40. La concentración de grupos aminos primarios se obtuvo mediante la ecuación:

$$
\left[\mathrm{NH}_{2}\right]=\mathrm{A} V / \varepsilon_{\max } l \cdot \mathrm{m}_{\text {muestra }}
$$

donde $\left[\mathrm{NH}_{2}\right]$ es la concentración de grupos amino libres ( $\mu \mathrm{mol} / \mathrm{g}$ piel seca), A es la absorbancia a $566 \mathrm{~nm}, V$ es el volumen de la solución de ninhidrina $(\mu \mathrm{L}), \mathrm{m}_{\text {muestra }}$ la masa de la muestra deshidratada (g), $\varepsilon_{\max }$ es la absortividad molar de los grupos amino en la reacción con ninhidrina $(1640 \mu \mathrm{L} / \mu \mathrm{mol} \mathrm{cm}, l$ longitud de la celda por donde pasa el 
haz $(1 \mathrm{~cm})$. El índice de reticulación (I de R) fue determinado mediante la ecuación:

I de $\mathrm{R}=\left(1-\left[\mathrm{NH}_{2}\right]_{\text {entrecruzado }} /\left[\mathrm{NH}_{2}\right]_{\text {fresso }}\right) \times 100$

donde $\left[\mathrm{NH}_{2}\right]_{\text {fresco }}$ es el contenido de grupos amino en la piel sin curtir, $\left[\mathrm{NH}_{2}\right]_{\text {entrecruzado }}$ es el contenido de grupos amino en el material curtido (tratado con el agente curtiente).

\section{Resultados y Discusion}

\section{Preparación del prepolímero}

En la Figura 1 se resumen las reacciones para la preparación de los prepolímeros.

Se sintetizaron prepolímeros a partir de los diisocianatos $\mathrm{H}_{12}$ MDI y HDI y polioles base poliéter de 1 y $2 \mathrm{kDa}$. En las Tablas 1 y 2 se resumen las condiciones de reacción, cantida-

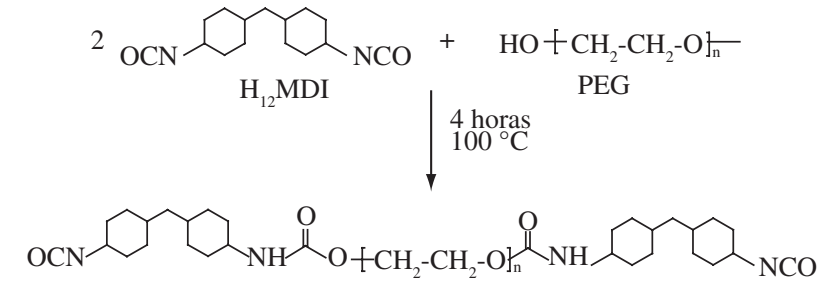

(a)

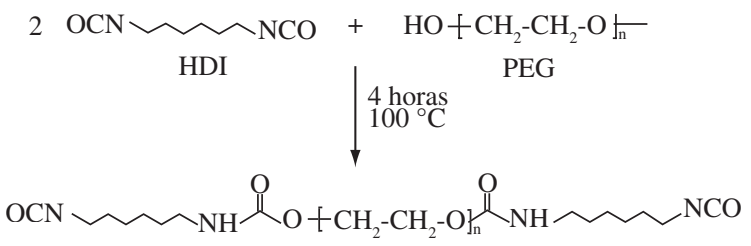

(b)

Figura 1. a) Reacción del $\mathrm{H}_{12} \mathrm{MDI}$ con poliol base óxido de etileno; y b) Reacción del HDI con poliol base óxido de etileno. des de reactivo y los resultados obtenidos por IR, en cuanto al tanto por ciento de NCO libre.

En las Figuras 2 y 3 se muestran los espectros infrarrojos al inicio y al final de la reacción, en los cuales se puede observar que al inicio no hay un pico intenso a $1715 \mathrm{~cm}^{-1}$, que es representativo del grupo carbonilo (prepolímero de poliuretano formado), mientras que el espectro que muestra el producto final de la reacción si presenta este pico.

Los resultados obtenidos en los experimentos nos indican que no hay mucha diferencia entre 2 y 4 horas de reacción ya que la determinación del contenido de NCO libre por espectroscopia IR presenta concentraciones similares.

\section{Bloqueo de los grupos isocianatos}

Con el propósito de incrementar la solubilidad y disminuir la toxicidad de los prepolímeros sintetizados, estos se hicieron

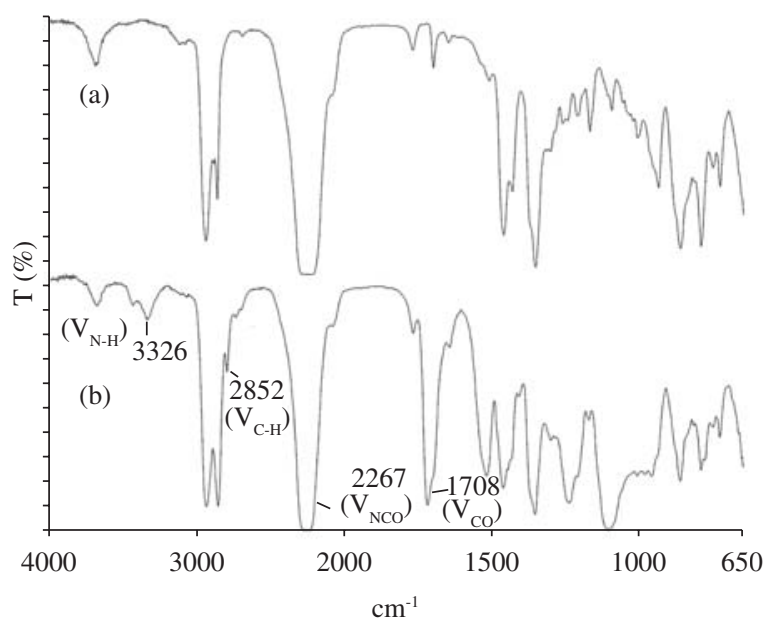

Figura 2. Espectros de IR de la mezcla de reacción de HDI + PEG1000; a) al inicio de la reacción; y b) después de 4 horas de reacción.

Tabla 1. Cantidades utilizadas de cada reactivo y su relación molar.

\begin{tabular}{lccccr}
\hline \multicolumn{1}{c}{ Reacción } & \multicolumn{2}{c}{ Cantidad de poliol } & \multicolumn{2}{c}{ Cantidad de isocianato } & \multirow{2}{*}{ Relación molar } \\
\cline { 2 - 4 } & Gramos & Moles & Mililitros & Moles & \\
\hline H $_{12}$ MDI + PEG-1000 & 2 & 0,002 & 2 & 0,0081 & $4: 1$ \\
H $_{12}$ MDI + PEG-2000 & 4 & 0,002 & 2 & 0,0081 & $4: 1$ \\
HDI + PEG-1000 & 2 & 0,002 & 2 & 0,0127 & $6: 1$ \\
HDI + PEG-2000 & 3 & 0,0015 & 2 & 0,0127 & $8,5: 1$ \\
\hline
\end{tabular}

Tabla 2. \% de isocianato libre después de cierto tiempo de reacción.

\begin{tabular}{cccc}
\hline \multicolumn{1}{c}{ Reacción } & Temperatura $\left({ }^{\circ} \mathbf{C}\right)$ & Tiempo de $\mathbf{R} \times \mathbf{n}(\mathbf{h})$ & Tanto por ciento de NCO libre \\
\hline \multirow{2}{*}{$\mathrm{H}_{12}$ MDI + PEG-1000 } & \multirow{2}{*}{100} & 2 & $36,5 \pm 0,62$ \\
\hline \multirow{2}{*}{$\mathrm{H}_{12}$ MDI + PEG-2000 } & \multirow{2}{*}{100} & 2 & $32,2 \pm 0,79$ \\
\hline \multirow{2}{*}{$\mathrm{HDI}+$ PEG-1000 } & \multirow{2}{*}{100} & 4 & $37,3 \pm 0,59$ \\
& & 2 & $35,3 \pm 0,08$ \\
\hline \multirow{2}{*}{$\mathrm{HDI}+$ PEG-2000 } & \multirow{2}{*}{100} & 4 & $36,7 \pm 0,04$ \\
& & 2 & $34,4 \pm 0,34$ \\
\hline
\end{tabular}

$\mathrm{n}=3 ; \mathrm{y} \pm$ Desviación estándar. 


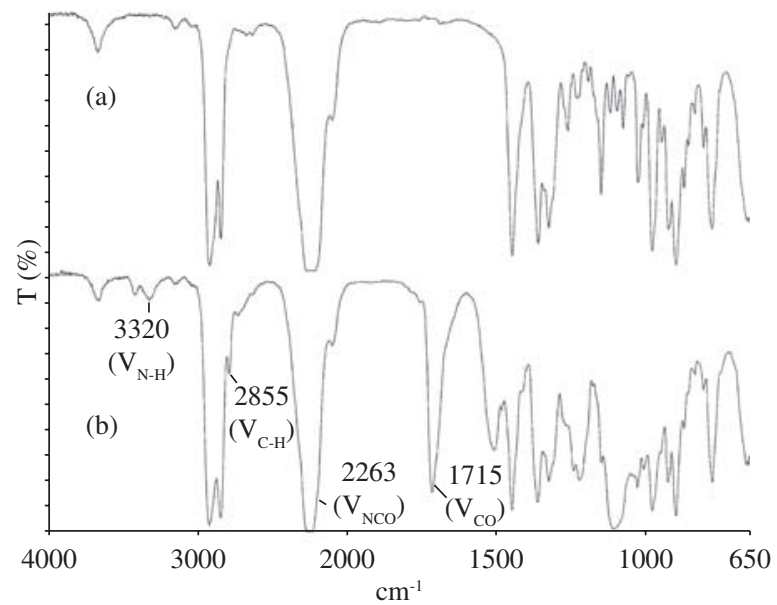

Figura 3. Espectros de IR de la mezcla de reacción de $\mathrm{H}_{12}$ MDI + PEG1000 a $100{ }^{\circ} \mathrm{C}$; a) al inicio de la reacción; y b) después de 4 horas de reacción.

reaccionar con una solución al $40 \%$ p/p de bisulfito o metabisulfito de sodio de sodio, en una proporción del doble de moles con respecto a la cantidad de isocianato presente en la reacción, 0,0162 mol. En la Figura 4 se resumen las reacciones para el bloqueo de los prepolímeros con bisulfito de sodio.

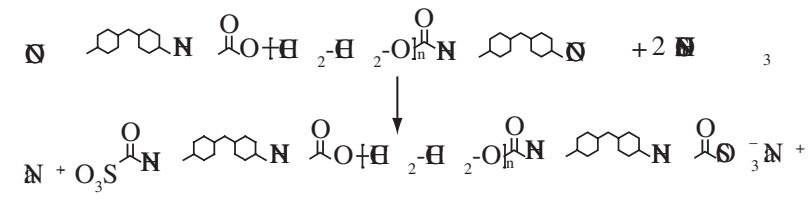

(a)

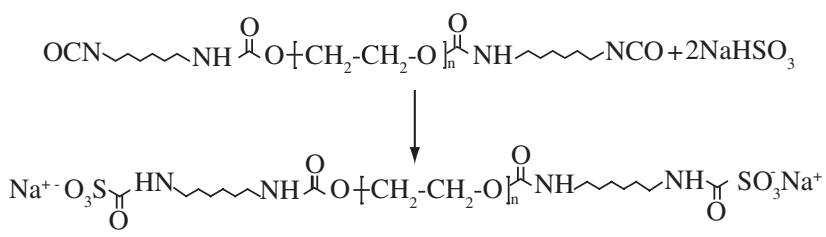

(b)

Figura 4. Reacción del bloqueo de los prepolímeros con bisulfito de sodio; a) Reacción con $\mathrm{H}_{12}$ MDI; y b) Reacción con HDI.

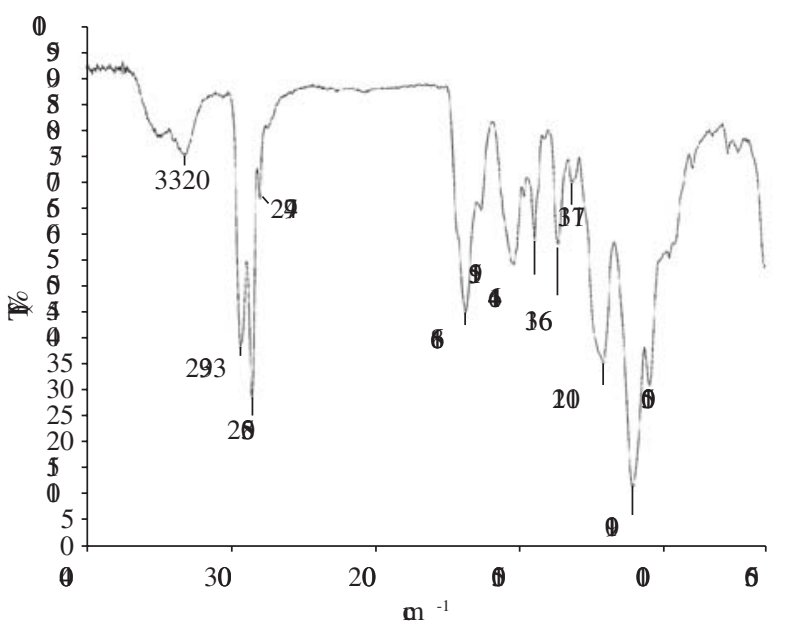

Figura 5. Espectro de IR del prepolímero sintetizado a partir de $\mathrm{H}_{12} \mathrm{MDI}$ y PEG 2000 después de 30 días de su síntesis.

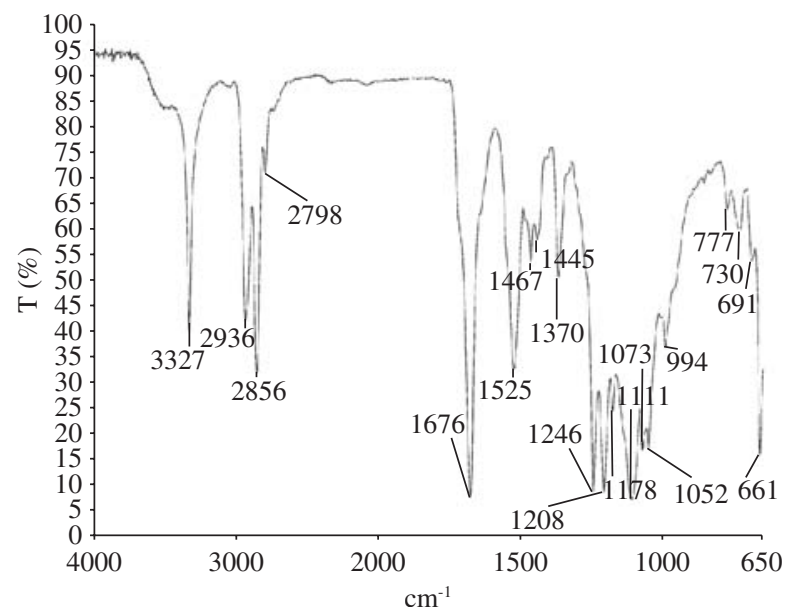

Figura 6. Espectro de IR del prepolímero sintetizado a partir de HDI y PEG1000 después de 30 días de su síntesis.

La estabilidad del prepolímero bloqueado fue analizada por espectroscopia IR. A una muestra del prepolímero depositada sobre el cristal de seleniuro de zinc del accesorio de ATR del espectrofotómetro de IR, se le realizó la exploración en un rango de 4000 a $650 \mathrm{~cm}^{-1}$ para obtener su espectro. La Figuras 5 y 6 muestran los espectros de IR para los prepolímeros obtenidos (después de 30 días de su síntesis), a partir de los diisocianatos $\mathrm{H}_{12}$ MDI y HDI, respectivamente. En ambos casos se puede apreciar la presencia de las bandas de absorción de las vibraciones $v_{\mathrm{N}-\mathrm{H}} \mathrm{y} \mathrm{v}_{\mathrm{NCO}}$ del poliuretano formado y la ausencia de la banda de absorción de la vibración $v_{\mathrm{NCO}}$ del grupo isocianato a $2267 \mathrm{~cm}^{-1}$ lo que nos indica que los prepolímeros se mantienen aún bloqueados.

\section{Evaluación del efecto curtiente de las DPU's}

Los resultados obtenidos a escala laboratorio, muestran que las DPU's presentan un efecto curtiente, que se favorece con el incremento en el porcentaje de dispersión utilizado. La Figura 7 muestra la posible interacción de la colágena de la piel con el agente curtiente. El efecto curtiente de las DPU's se basa en su reactividad con los grupos amino presentes en la piel. La lisina es uno de los aminoácidos que tienen grupos amino libres en la colágena. Las DPU's necesitan que éstos se encuentren en su forma no protonada. En condiciones ligeramente ácidas, son protonados, por lo que no podrían reaccionar con las DPU's. Un pH neutro o ligeramente alcalino favorecerá la forma no protonada ${ }^{[9]}$ y con ello la interacción con las DPU's (Figura 8). El no requerir un $\mathrm{pH}$ ácido para realizar la curtición nos evita la etapa de picle en el proceso de curtido, indispensable para curticiones con sales de cromo y glutaraldehído ${ }^{[9]}$. En la Figura 9 se presentan los resultados de la temperatura de desnaturalización e índice de reticulación de las pieles procesadas.

El efecto curtiente de la piel con glutaraldehído (GA) alcanza una temperatura de desnaturalización de la piel de $\mathrm{T}=83{ }^{\circ} \mathrm{C}-87^{\circ} \mathrm{C}$ y un índice de reticulación $\mathrm{I}$ de $\mathrm{R}=80-82$. $\mathrm{El}$ tratamiento de la piel con las DPU's ha alcanzado un valor de $\mathrm{T}=84{ }^{\circ} \mathrm{C}$ y un índice de reticulación, I de $\mathrm{R}=72$, muy cercano al obtenido con GA. 


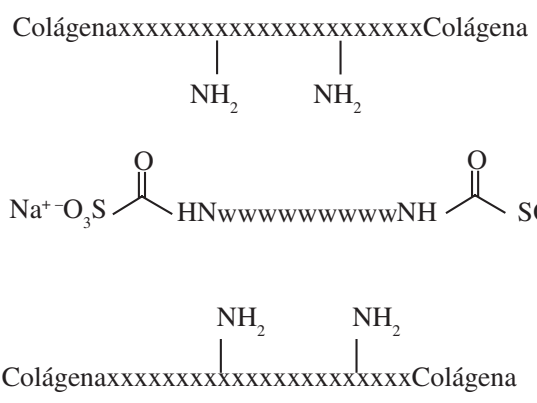

Figura 7. Curtición con dispersiones acuosas de poliuretano bloqueado (DPU's).

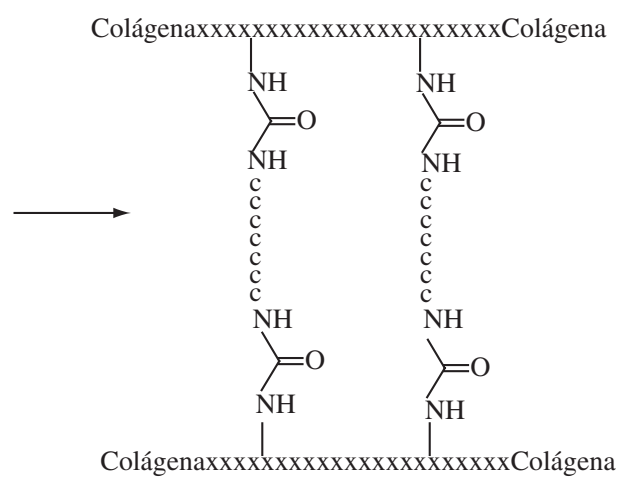

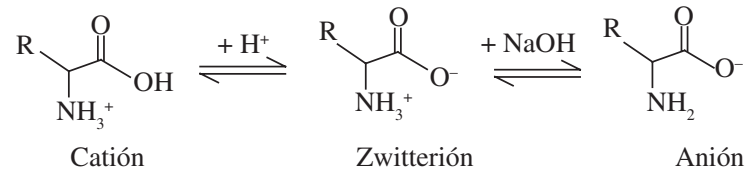

Carga neta $=0$

Figura 8. Estado de los grupos amino a diferente $\mathrm{pH}$.

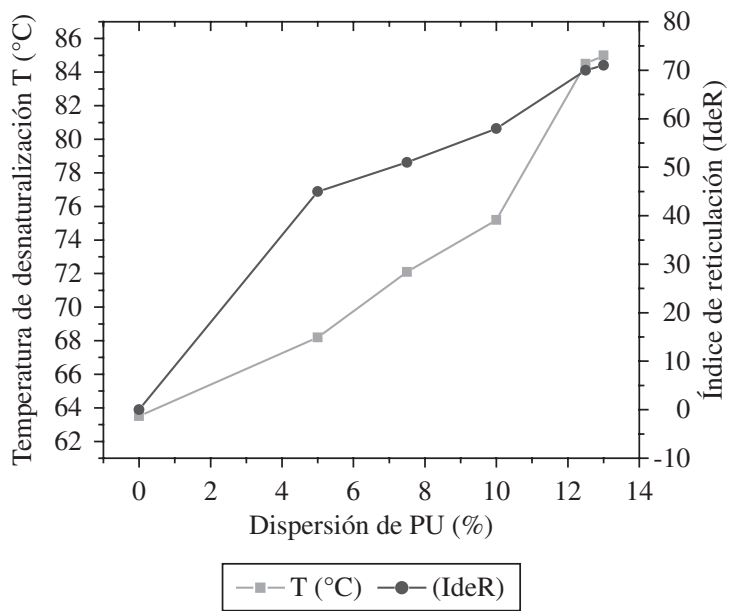

Figura 9. Efecto del porcentaje de DPU's utilizado en la curtición sobre la Temperatura de desnaturalización e índice de reticulación de la piel.

\section{Conclusiones}

Fue posible realizar la síntesis de dispersiones acuosas de poliuretano basadas en prepolímeros a partir de la reacción de los diisocianatos alifáticos $\mathrm{H}_{12}$ MDI y HDI con polioles base poliéter óxido de etileno de peso molecular 1 y $2 \mathrm{kDa}$. El análisis por espectroscopía IR de las dispersiones permitió confirmar el bloqueo de los grupos isocianatos libres, así como su estabilidad.

Los resultados del estudio de curtición a nivel laboratorio muestran que las DPU's presentan un efecto curtiente. Se logró un incremento en la temperatura de desnaturalización de 65 a $84{ }^{\circ} \mathrm{C}$, lo que demuestra la interacción de la colágena de la piel con las DPU's, que se refleja en el índice de reticulación (un valor de 72). Una las ventajas que presenta este tipo de agente de reticulación es su efecto curtiente a $\mathrm{pH}$ neutros ó ligeramente alcalinos lo que nos evita la etapa de picle en la curtición.
El empleo de un aceite sulfitado y un recurtiente fenólico en la curtición mejoran las propiedades de flexibilidad y cuerpo del cuero obtenido.

Este tipo de dispersiones acuosas de poliuretano puede ser un excelente y viable agente curtiente como alternativa al glutaraldehído, que actualmente se utiliza en la producción de cuero para la industria automotriz.

\section{Agradecimientos}

Los autores agradecen al Consejo de Ciencia y Tecnología del Estado de Guanajuato (CONCyTEG) por el apoyo económico para la realización de este proyecto de investigación. Asimismo, al Dr. Roberto Zitzumbo Guzmán del Centro de Innovación Aplicada en Tecnologías Competitivas CIATEC por los estudios de Calorimetría Diferencial de Barrido (DSC).

\section{Referencias}

1. (a) Fein, E. H., Harris, E. H., Naghki, J. \& Filachione, E. M. - Journal of the American Leather Chemists Association JALCA, 54, p.488. (1959). (b) Seligsberger, L., Sadlier, C. Journal of the American Leather Chemists Association JALCA, 52, p.2. (1957).

2. Petersen, S. - Justus Liebig Ann. Chem., 562, 205 (1948).

3. Traubel, H. - Journal of the American Leather Chemists Association JALCA, 100, p.304. (2005).

4. Kenyon, W. O., Smith D. A. \& Taylor, E. W. U.S. - Patent 3, 220, p.864 (1965).

5. Cater, C. J. - Soc. Leather Trades Chem., 49, p.455 (1965).

6. Milligan, B.; Buecheler, P. - Journal of the American Leather Chemists Association JALCA, 77, p.70 (1982).

7. Traubel, H. \& Reiff, H. - U.S. Patent 5, 618, p.317 (1997).

8. Traubel, H. - The Sirolan-BAP-process "Polyurethanes for Textiles, Paper and Leather", in Günter Oertel, "Polyurethane Handbook", Munich., 575 (1994).

9. Heidemann, E. - Fundamentals of Leather Manufacture. Darmstadt, p.194 (1993).

Enviado 10/07/07

Reenviado: $17 / 11 / 07$

Aceito: $21 / 11 / 07$ 